\title{
Online Finger Gesture Recognition Using Surface Electromyography Signals*
}

\author{
Qiang Li, Bo Li \\ School of Information Engineering, Southwest University of Science and Technology, Mianyang, China. \\ Email: liqiangsir@gmail.com, leebowolf@gmail.com
}

Received January $9^{\text {th }}$, 2013; revised February $11^{\text {th }}$, 2013; accepted February $19^{\text {th }}, 2013$

Copyright (C) 2013 Qiang Li, Bo Li. This is an open access article distributed under the Creative Commons Attribution License, which permits unrestricted use, distribution, and reproduction in any medium, provided the original work is properly cited.

\begin{abstract}
The analysis on the online finger gesture recognition using multi-channel sEMG signals was explored in this paper. Nine types of gestures were applied to be identified, involving six kinds of numerical finger gestures and three kinds of hand gestures. The time domain parameters were extracted to be the features. And then, the probabilistic neural network was utilized to classify the proposed gestures with the extracted features. The experimental results showed that most of gestures could acquire the acceptable classification performance and a few elaborate gestures were hard to acquire the effective identification.
\end{abstract}

Keywords: Finger Gesture; sEMG Signal; Online; Recognition

\section{Introduction}

The gesture recognition is an important technique that can provide intelligent and flexible ways to implement the robotic and rehabilitation equipments for disable people or amputees. When the surface electrodes are placed on the muscle skin, the muscular dynamic contraction process can be described by the surface electromyography (sEMG) signals. But the measured signal is interfered by various factors, such as, physiological noise, motion artifact, electronic devices, etc., so, it is difficult for the analysis and application of sEMG signal directly. By reason of the non-destructive collection advantage, the gesture recognition manner based on the sEMG signal is realizable and more acceptable.

The pattern recognition processing is necessary to identify the different gestures using sEMG signal, and many algorithms have been applied [1]. The data segmentation method was used to acquire the data segments about actions. Moving average, approximated generalized likelihood ratio, nonlinear Teager-Kaiser energy operator and many other methods were used to detect the muscle activity [2]. The feature extraction method was utilized to obtain the parameters which could represent the action sEMG signals. The parameters about time domain, frequency domain and time-frequency domain were

*Scientific Research Fund of Sichuan Provincial Education Department (12ZA185) and Doctor Foundation of SWUST (08zx0110). analyzed, they could be divided into the structural and phenomenological approaches. Especially, the time-frequency features, such as short time Fourier transform and wavelet transform, were complicated and high dimensional, so, the dimensionality reduction of the feature vector was required, and the classifier speed could be increased without loss of classification accuracy, and the principle component analysis (PCA) was utilized to accomplish this purpose [3,4]. And, the autoregressive (AR) coefficients [5] and mother wavelet matrix [6] could be adopted. Then, the pattern classification was employed. Neural network, fuzzy theory, probabilistic approach, support vector machine and grey relational analysis based classifier were adopted to achieve the recognition results $[1,7,8]$.

Additionally, the effect of electrode displacements about classification accuracy was also explored [9]. Multiple sensors were utilized to help the gesture identification, including video and accelerometer [10]. But most of the current work focused on the gestures of wrist and arm. Recently, the study of finger gestures was concerned, it could acquire the elaborate action recognition and enlarge the application of robotic prosthesis. Typically, the flexion and extension movements of each fingers, finger keypress gestures and grasp actions had been analyzed [1113].

In this work, the finger gesture recognition was discussed using multi-channel sEMG signals. The six kinds 
of numerical gestures of one to six and three kinds of hand gestures were explored. And the time domain parameters were extracted to be the features. Then the probabilistic neural network (PNN) was employed to do the classification.

\section{Material and Methodology}

\subsection{Signal Measurement and Gestures}

Multi-channel sEMG signals measurement system was designed, and four channel signals were applied. The electrode configuration was adopted to be bipolar, and the electrodes were placed on the forearm muscles of healthy male volunteer. The illustration of electrode placements was showed in Figure 1.

Nine types of gestures were showed in Figure 2. The Figures 2(a)-(f) represented the six kinds of numerical gestures, and they were one, two, three, four, five and six. Then, the Figure 2(g) represented the flexion of wrist, Figure 2(h) represented the extension of wrist, and Figure 2(i) represented making a fist.

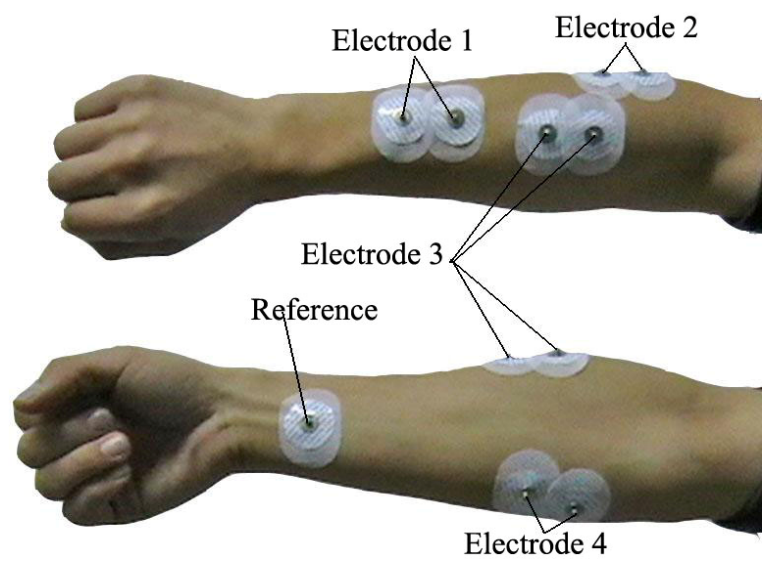

Figure 1. Illustration of electrode placements.

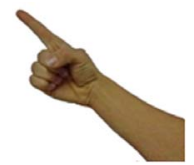

(a) $\mathrm{ONE}$

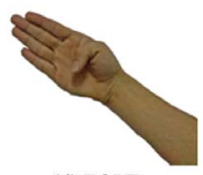

(d) FOUR

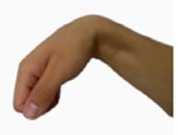

(g) FLWR

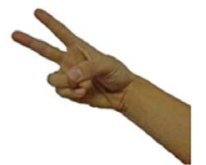

(b) TWO

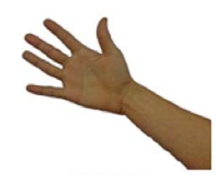

(e) FIVE

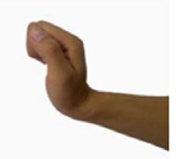

(h) EXWR

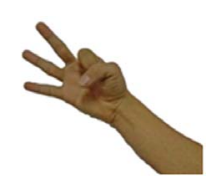

(c) THREE

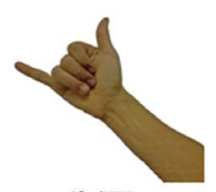

(f) SIX

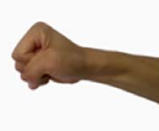

(i) MAFT
Figure 2. Nine types of gestures.
Using the data acquisition card of PCI-6221 within the computer, the four channel sEMG signals could be collected. For the improvement of signal quality, the bandpass filter, spectrum interpolation technique [14] and wavelet analysis method were implemented and applied for the noise signal reduction.

\subsection{Feature Extraction}

To acquire the data segmentation from the gesture sEMG signal, the moving average algorithm was employed [5, $15]$.

With the segmented data, the feature parameters of multi-channel sEMG signals were evaluated. Three parameters were utilized and expressed as below $[5,8,11]$.

1) Mean absolute value

$$
\operatorname{Mav}=\frac{1}{N} \sum_{i=1}^{N}\left|x_{i}\right|
$$

2) Variance

$$
\operatorname{Var}=\frac{1}{N-1} \sum_{i=1}^{N} x_{i}^{2}
$$

3) AR coefficients

$$
x_{i}=\sum_{k=1}^{p} a_{k} x_{i-k}+e_{i}
$$

where, $x$ represented the data segmentation of sEMG signals and $N$ was the total number of the data segmentation, $p$ is the order of the AR model, $e$ is a random noise term, and then, $a_{k}(k=1, \cdots, p)$ represented the AR coefficients. Finally, with the four channel sEMG signals, the three above-mentioned feature parameters of each channel were extracted and used to create the feature vector.

According to the extracted features of four-channel signals, the space information about the activities of different muscles could be expressed, and it could help the classification analysis. More, the adopted features were computed expediently, and it could facilitate the online processing.

\subsection{Recognition Method}

The PNN is a nonlinear classifier that the Bayesian decision criterion and the feed-forward computation are used. It is fit for the online classification. The training process of PNN is fast and its processing is parallel.

The structure of PNN has four layers, including input layer, pattern layer, summation layer and decision layer, and it is illustrated in Figure 3. The input layer receives the input feature vector $\boldsymbol{V}=\left[\begin{array}{llll}v_{1} & v_{2} & \cdots & v_{k}\end{array}\right]$, and $k$ is the number of feature values. And then, the pattern layer calculates the computation of input vector and weight vector, and the output of activation level can be obtained 
by a nonlinear operation of $g(\cdot)$ function. The parameter $m_{i}(i=1, \cdots, n)$ denotes the number of training vector in the $i$-th class, and $n$ is the total number of the classes. The summation layer calculates the summation according to the output parameters of pattern layer and multiplication with the weight factors, and the parameter $f_{i}(i=1, \cdots, n)$ represents the summation of probability densities in the $i$-th class. Finally, the decision layer acquires the output of classification result which the largest value of summation layer can be selected to be the solution $[16,17]$. With the feature extraction and processing

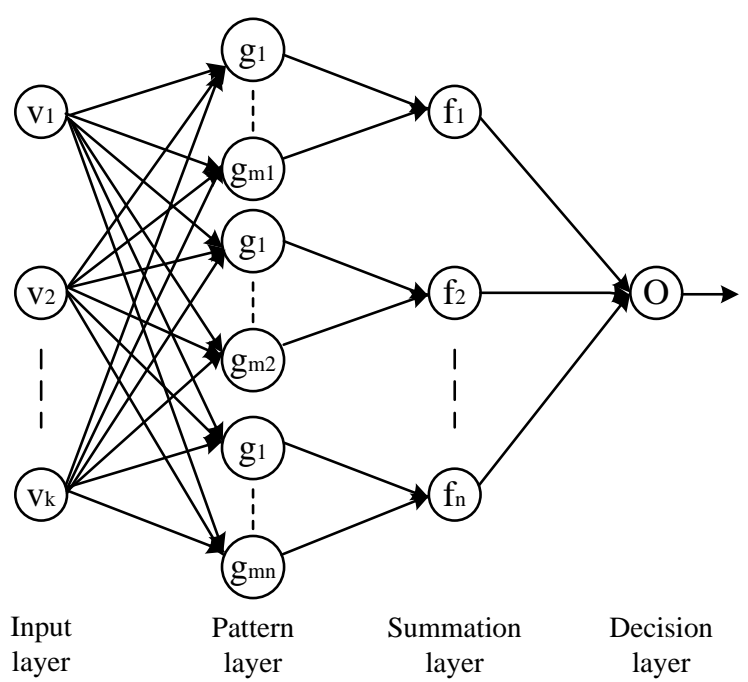

Figure 3. Illustration of probabilistic neural network structure. of PNN classifier, the proposed gestures could be identified.

\section{Experimental Analysis}

By utilizing the designed sEMG signal measurement circuit and proposed data acquisition card, the experimental system of four channel signals collection and online gesture recognition was implemented with visual $\mathrm{c}^{++}$. The designed interface of this experimental system was illustrated in Figure 4. In this interface, the four channel sEMG signals were showed, the corresponding recognized result was demonstrated as the pre-set gesture picture, and the sampling rate was set to be $1 \mathrm{kHz}$.

In the experiment, 4-order AR coefficients were adopted to be the features with mean absolute value and variance. At the training stage, each gesture was set to repeat 20 times to train the neural network offline. At the testing stage, the 20 times of each gesture were also utilized to accomplish the recognition online, and the experimental results were showed in Table 1. It could be found that the finger gestures of "TWO" and "FOUR" were not identified successfully because their recognized rates were only $45 \%$ and $55 \%$, the finger gesture of "SIX" was difficult to be identified, and the others could be considered as the effective recognition results because their recognized rates were equal to and above $70 \%$. The online experiments showed that the online recognition system could work satisfactorily, but some finger gestures were hard to be distinguished because the features of these elaborate actions were very close.

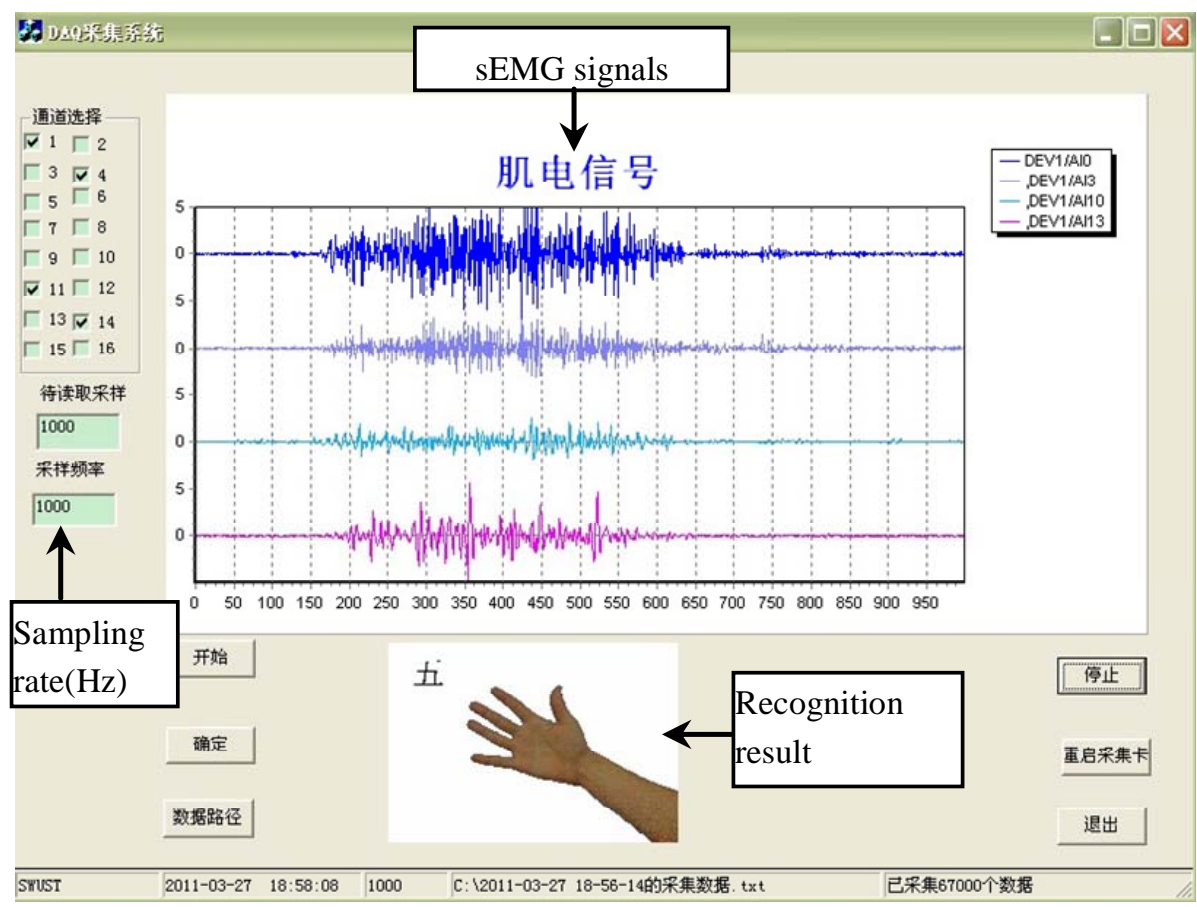

Figure 4. Interface of designed sEMG signals measurement and recognition. 
Table 1. Online experimental results of gesture recognition.

\begin{tabular}{|c|c|c|c|c|c|c|c|c|c|c|}
\hline \multirow{2}{*}{ Actions } & \multicolumn{9}{|c|}{ Recognized number in the online experiment } & \multirow{2}{*}{$\begin{array}{c}\text { Recognized } \\
\text { rate }\end{array}$} \\
\hline & ONE & TWO & THREE & FOUR & FIVE & SIX & FLWR & EXWR & MAFT & \\
\hline ONE & 18 & 0 & 0 & 0 & 0 & 0 & 1 & 0 & 1 & $90 \%$ \\
\hline TWO & 3 & 9 & 8 & 0 & 0 & 0 & 0 & 0 & 0 & $45 \%$ \\
\hline THREE & 0 & 0 & 16 & 1 & 0 & 0 & 3 & 0 & 0 & $80 \%$ \\
\hline FOUR & 0 & 0 & 7 & 11 & 2 & 0 & 0 & 0 & 0 & $55 \%$ \\
\hline FIVE & 2 & 0 & 0 & 0 & 16 & 0 & 0 & 2 & 0 & $80 \%$ \\
\hline SIX & 4 & 0 & 0 & 0 & 0 & 13 & 0 & 3 & 0 & $65 \%$ \\
\hline FLWR & 4 & 0 & 0 & 0 & 0 & 0 & 16 & 0 & 0 & $80 \%$ \\
\hline EXWR & 6 & 0 & 0 & 0 & 0 & 0 & 0 & 14 & 0 & $70 \%$ \\
\hline MAFT & 1 & 0 & 0 & 0 & 0 & 0 & 0 & 4 & 15 & $75 \%$ \\
\hline
\end{tabular}

\section{Conclusion}

An experimental system of sEMG signals measurement and online gesture recognition was implemented. By the proposed method, some effective recognized results were acquired, but the elaborate actions were hard to be classified because of the indistinguishable features. So, much work about signal analysis and online classification method needed to be improved.

\section{REFERENCES}

[1] M. A. Oskoei and H. Hu, "Myoelectric Control Systems-A Survey," Biomedical Signal Processing and Control, Vol. 2, No. 4, 2007, pp. 275-294. doi:10.1016/j.bspc.2007.07.009

[2] X. Y. Li and A. S. Aruin, "Muscle Activity Onset Time Detection Using Teager-Kaiser Energy Operator," 27th Annual Conference of IEEE Engineering in Medicine and Biology, Shanghai, 2005, pp. 7549-7552.

[3] J. U. Chu, I. Moon and M. S. Mun, “A Real-Time EMG Pattern Recognition System Based on Linear-Nonlinear Feature Projection for a Multifunction Myoelectric Hand," IEEE Transactions on Biomedical Engineering, Vol. 53, No. 11, 2006, pp. 2232-2239. doi:10.1109/TBME.2006.883695

[4] M. Khezri and M. Jahed, "An Exploratory Study to Design a Novel Hand Movement Identification System," Computers in Biology and Medicine, Vol. 39, No. 5, 2009, pp. 433-442. doi:10.1016/j.compbiomed.2009.02.001

[5] X. Chen, Q. Li, J. H. Yang and V. Lantz, “Test-Retest Repeatability of Surface Electromyography Measurement for Hand Gesture," 2nd International Conference on Bioinformatics and Biomedical Engineering, Shanghai, China, 2008, pp. 1923-1926.

[6] J. Rafiee, M. A. Rafiee, F. Yavari and M. P. Schoen, "Feature Extraction of Forearm EMG Signals for Prosthetics," Expert Systems with Applications, Vol. 38, No. 4, 2011, pp. 4058-4067. doi:10.1016/j.eswa.2010.09.068

[7] M. A. Oskoei and H. Hu, "Support Vector MachineBased Classification Scheme for Myoelectric Control Applied to Upper Limb," IEEE Transactions on Biomedical Engineering, Vol. 55, No. 8, 2008, pp. 1956-
1965. doi:10.1109/TBME.2008.919734

[8] Y. C. Du, C. H. Lin, L. Y. Shyu and T. Chen, "Portable Hand Motion Classifier for Multi-Channel Surface Electromyography Recognition Using Grey Relational Analysis,” Expert Systems with Applications, Vol. 37, No. 6, 2010, pp. 4283-4291. doi:10.1016/j.eswa.2009.11.072

[9] L. Hargrove, K. Englehart and B. Hudgins, “A Training Strategy to Reduce Classification Degradation Due to Electrode Displacements in Pattern Recognition Based Myoelectric Control," Biomedical Signal Processing and Control, Vol. 3, No. 2, 2008, pp. 175-180. doi:10.1016/j.bspc.2007.11.005

[10] W. H. Wang, X. Chen, P. Yang, Y. Li and J. H. Yang, "Chinese Sign Language Recognition Based on Multiple Sensors Information Detection and Fusion," Chinese Journal of Biomedical Engineering, Vol. 29, No. 5, 2010, pp. 665-671.

[11] F. V. G. Tenore, A. Ramos, A. Fahmy, S. Acharya, R. Etienne-Cummings and N. V. Thakor, "Decoding of Individuated Finger Movements Using Surface Electromyography,” IEEE Transactions on Biomedical Engineering, Vol. 56, No. 5, 2009, pp. 1427-1434. doi:10.1109/TBME.2008.2005485

[12] C. Cipriani, C. Antfolk, M. Controzzi, G. Lundborg, B. Rosen, M. C. Carrozza, et al., "Online Myoelectric Control of a Dexterous Hand Prosthesis by Transradial Amputees," IEEE Transactions on Neural Systems and Rehabilitation Engineering, Vol. 19, No. 3, 2011, pp. 260270. doi:10.1109/TNSRE.2011.2108667

[13] J. Cheng, X. Chen, Z. Y. Lu, X. Zhang and Z. Y. Zhao, "Research on Finger Key-Press Gesture Recognition Based on Surface Electromyographic Signals,” Journal of Biomedical Engineering, Vol. 28, No. 2, 2011, pp. 352-356.

[14] D. T. Mewett, K. J. Reynolds and H. Nazeran, "Reducing Power Line Interference in Digitised Electromyogram Recordings by Spectrum Interpolation,” Medical \& Biological Engineering \& Computing, Vol. 42, No. 4, 2004, pp. 524-531. doi:10.1007/BF02350994

[15] M. Lei and Z. Z. Wang, “A Method for Real-Time Pickup Action Signal," Chinese Journal of Medical Instrumentation, Vol. 24, No. 4, 2000, pp. 200-202.

[16] D. F. Specht, "Probabilistic Neural Networks," Neural Networks, Vol. 3, No. 1, 1990, pp. 109-118. 
doi:10.1016/0893-6080(90)90049-Q

[17] X. J. Shang, Y. T. Tian, Y. Li and L. G. Wang, "Recognition of Gestures and Movements Based on MPNN," Jour- nal of Jilin University (Information Science Edition), Vol. 28, No. 5, 2010, pp. 459-466. 